\title{
Periferias espontáneas de Ciudad Bolívar-Bogotá: vista desde la perspectiva fractal. Abordaje metodológico
}

\author{
Spontaneous Peripheries of Ciudad Bolívar-Bogotá: view from the fractal \\ perspective. Methodological approach
}

\author{
Alejandro Guerrero Torrenegra, ${ }^{1}$ M.Sc. \\ ${ }^{1}$ Universidad de Bogotá Jorge Tadeo Lozano, Bogotá, Colombia.
}

Recibido: 27 de marzo de 2016. Aceptado: 17 de mayo de 2016.

Guerrero, A. (2016) Periferias espontáneas de Ciudad Bolívar-Bogotá: vista desde la perspectiva fractal. Abordaje metodológico. Procesos urbanos Número 3, Ene-Dic. 89-98. Doi:10.21892/2422085X.269

\section{RESUMEN}

Las periferias internas espontáneas de América Latina se caracterizan por sus genes tipológicos informales-invasiones, que identifican a los tejidos informales que se producen como consecuencia de las construcciones y reconstrucciones auto-gestionadas. El objetivo principal de la investigación fue identificar la existencia de patrones o códigos fractales en las manzanas de la periferia interna espontánea de las UPZ, de la localidad de Ciudad Bolívar de Bogotá. La investigación es descriptiva y analítica, sigue pautas fenomenológicas, con matemática (no lineales) y geometría fractal. El resultado originó otra manera de aproximación al problema, en el que la morfología urbana guarda una relación con los tamaños y sus dimensiones: longitud, superficie y volumen, variables que conforman el plan, el diseño o la renovación urbana.

Palabras clave: Periferias urbanas, fractales, patrones fractales

\begin{abstract}
The spontaneous internal peripheries of Latin America are characterized by their informal type-invasion genes, which identify informal fabrics that occur because of self-managed constructions and reconstructions. The main objective of the research was to identify the existence of patterns or fractal codes in the blocks of the spontaneous internal periphery of UPZ, in the town of Ciudad Bolívar, Bogotá. The research is descriptive and analytical, follows phenomenological guidelines, with mathematics (non-linear) and fractal geometry. The result originated another way of approaching the problem, in which the urban morphology is related to the sizes and their dimensions: length, surface and volume, variables that make up the plan, the design or the urban renewal.
\end{abstract}

Key words: Urban peripheries, fractals, fractal patterns 


\section{INTRODUCCIÓN}

La periferia se identifica por ser fragmentos con genes tipológicos informalesespontáneos, que están dentro de un escenario de las ciudades difusas, identificados por la profundización de su insostenibilidad económica, política, ambiental, social, urbana, y cultural. Los genes tipológicos informales están en mutación continua que se refleja en el caos urbano y desequilibrio entre los humanos y la naturaleza, la desigualdad, la complejidad, la globalización, y la gobernanza (Ferrer 2012). A medida que los seres humanos avanzan la complejidad aumenta y se introducen mecanismos descentralizadores y de participación pública los cuales colapsan.

La distribución de los tamaños de la ciudad está relacionada en forma directa con los espacios urbanos libres, áreas residenciales y comerciales, urbanizaciones espontáneas, áreas verdes, calles, edificaciones; al respecto, Salingaros (2005), expresa que, con la llegada de la liberación del paradigma de "definir escala", se originan los fractales en cualquier escala hasta el infinito. La vida de la ciudad está identificada por la matriz de conexión y las subestructuras urbanas, ya que la geometría puede establecer el fortalecimiento o debilitación del movimiento e interacción de las personas.

De allí que, para la investigación, se comienza con la caracterización de elementos que conforman la ciudad: sendas, bordes, barrios, nodos y mojones, Lynch (2012). Este proceso se realizó con la lectura del plano ${ }^{1}$ de la ciudad y la aplicación de la observación en realidad social, urbana, política, cultural, ambiental, económica del contexto en estudio, para garantizar el tamaño y figura de la ciudad, de los municipios y la distribución de las localidades $^{2}$, para visualizar: el tejido, la trama, las manzanas o estructura parcelaria, que obedece a la arquitectura y la morfología urbana, Borie, Micheloni, Pinon (2008).

\footnotetext{
1 Plano. Representación esquemática, en dos dimensiones y a determinada escala, de un terreno, una población, una máquina, una construcción (DRAE 2014).

2 Localidades: subdivisión administrativa de la ciudad, cada localidad cuenta con un alcalde local que es el intermedio entre los ciudadanos y la administración distrital (http://www.bogotacomovamos.org/localidades/barrios-unidos).

La lectura del plano urbano nos demuestra la morfología de la estructura urbana, el crecimiento y la mutación del espacio en tiempo y forma de apropiación de las manzanas y parcelas. La figura presente en el plano de la ciudad, está unida al tamaño de la urbe, seguido en orden decreciente, por el municipio, el tamaño de la localidad, la UPZ, las manzanas y parcelas.

Salingaros (2005) citado por Pineda (2005), dice que la distribución de los tamaños es continua y hasta el infinito. Estos factores se reflejan cuando combinan con la regla de las escalas, es el momento apropiado para que la distribución de los tamaños se haga más discreta.

La presente investigación, desarrolló un análisis para la identificación de las figuras de las manzanas, con una morfología geométrica igual, pero con distinta superficie, con el parámetro de presunta semejanza ${ }^{3}$, determinando una relación de proporcionalidad entre sus lados.

La existencia de la auto-relación puede determinar la presencia de patrón fractal entre la morfología del tejido urbano, situación que se coteja con análisis complementario. No existe ley mecánica para los sistemas dinámicos caóticos que generen formas o sistemas iguales, pero sí, procesos urbanos auto-semejantes y los que responden a procesos culturales, económicos, y los sociales de homogenización y de estandarización.

Estos procesos se originan en un lugar geográfico específico, el cual está conformado en características topográficas, geológicas, climáticas y en orientación que no establecen, pero sí determinan la evolución urbana. De esta manera los tamaños (áreas) de las zonas, barrios, manzanas, predios, y sus genes tipológicos (forma de ocupación), pueden responder a un patrón ordenador no perceptible. En el caso de esta investigación solo se abordó el estudio de manzana, para de esta manera identificar los patrones o códigos fractales.

\footnotetext{
3 Semejante: Dos figuras distintas solo por su tamaño y cuyas partes guardan todas respectivamente la misma proporción. (DRAE, 2014)
} 
Guerrero, A. - Periferias espontáneas de Ciudad Bolívar, Bogotá.

\section{METODOLOGÍA}

Investigación es descriptiva y analítica. Para la realización de esta exploración, se utilizó el método fenomenológico (busca la compresión y demostración de la esencia), partiendo de la premisa que el 'sistema de la razón se despliega" (Leal (2000, p.52). Lo que orienta a realizar el abordaje de esta realidad partiendo de la observación propia del investigador de un contexto en estudio y relacionándolo con aspectos sociales, económicos, culturales, políticos y urbanos desde la percepción de las personas cimentado en la experiencia interna de cada individuo (sentimientos, recuerdos, vivencias).

Etapa 1, se apodera de las teorías clásicas para cotejarlas con las vigentes, apoyándose en los principios de las nuevas ciencias: teoría del caos, la incertidumbre, la complejidad, las figuras geométricas y los fractales, lo que permitió la aplicación del lenguaje y ecuaciones matemáticas sencillas, que identifican a los sistemas no lineales, para convertirlos; en morfologías urbanas irregulares y espontáneas. Se ensayó, alterando el proceso de "teoría-práctica" por el de "búsqueda-observación-reflexión, exploración y construcción" (Pineda, 2005).

Etapa 2, parte analítica está fundamentada en el método descriptivo aplicado en las fases del proceso, mediante los conocimientos forjados por la observación y la experiencia cotidiana, también es alimentada por las vivencias de los diferentes espacios urbanos, por parte de sus habitantes, ocasionando la identificación de propiedades, características y rasgos, de las manifestaciones urbanas analizadas.

Etapa 3, se construyeron los procesos y métodos, que permiten transportar las imágenes abstractas, aplicando fórmulas matemáticas que ayudan a expresar la relación variable- parámetro-variable, en la construcción del conocimiento, para luego convertirlas en formas geométricas para la definición del tejido urbano espontáneo, afines al comportamiento humano.

Las herramientas metodológicas: La revisión del material cartográfico, las cartillas de las UPZ de la localidad de Ciudad Bolívar, artículos relacionados con los asentamientos espontáneos en la ciudad de Bogotá, planos digitales. Para leer la morfología de las UPZ, se utilizaron los planos digitales de la página de internet portal de mapas Bogotá ( mapas.bogota.gov.co/portalmapas/) de la Unidad Administrativa Especial de Catastro Distrital, la cual permite ver el plano a diferentes escalas que ayudan a comprender las dimensiones y determinar el tamaño total del supersector, las UPZ, el tamaño de las manzanas y parcelas; identificar la estructura urbana, la senda, los nodos, el tejido, los hitos, y la localización de equipamientos; los datos resultantes de los planos digitales se confrontaron in situ.

En relación con la inserción teórica, se fundamentó con base en las referencias de algunos autores, cuya práctica se integró a la investigación en la medida que se necesitara. Los autores fueron Moisset (1998), Morín (2011; 2005), Ugas (2012; 2010), Pineda (2005), Lynch (2012), Borie, Micheloni, Pinon (2008), y Salingaros (2005).

\section{ESTUDIO DE CASO}

En la investigación se toma como referencia la ciudad de Bogotá que fue fundada en 1538 , identificada por su carácter colonial con rasgos formales de la cultura española. La localidad de Ciudad Bolívar, está ubicada al sur de la ciudad de Bogotá y limita, al norte con la localidad de Bosa; al sur con la localidad de Usme, al oriente con la localidad de Tunjuelito y Usme y al occidente con el municipio de Soacha, integrada por 8 UPZ, con una extensión urbana de 3.391 hectáreas (ha), y por 326 barrios, el 90\% es montañoso (topografía inclinada).

Se escogen (3) unidades de planeación zonal (UPZ) para conformar un supersector, la UPZ no66, San Francisco, con uso residencial de urbanización incompleta, con una extensión de 179 (ha), está integrada por 21 barrios. UPZ 69 Ismael Perdomo, es de uso residencial de urbanización incompleta más extensa, con 559 (ha) cuenta con zona industrial y conformada por 91 barrios. UPZ 70 Jerusalén, con uso del suelo residencial 
de urbanización incompleta ${ }^{4}$ y tiene una extensión de 537 hectáreas, conformada por 907 manzanas y 15 barrios (Secretaria de planeación 2009).

Zona San Francisco, ocupa el 13\% del territorio del supersector, ubicada en el cuadrante entre la av. Ciudad de Villavicencio y av. Tunjuelito, en sentido norte-este, desde la av. Boyacá y la carrera 18Z, en el sentido este-sur, con la diagonal 69, en sentido sur-oeste, y con la av. Mariscal de Sucre, en sentido oeste-norte.

Esta UPZ San francisco, está fragmentada en dos pedazos como consecuencia de la quebrada Limas, que hace un recorrido en sentido suroccidente-nor-oriente con un alto problema de contaminación en aguas; conforma manzanas con morfología fractal producto de la naturaleza (relativo a la forma del cauce), con lados ondulados e inclinados, las cuales se conformaron en ambos lados del cuerpo de agua infringiendo la ronda hidráulica ${ }^{5}$ como zona protectora de $30 \mathrm{~m}$. de ancho para las dos franjas, (Plan de Gestión Ambiental del Distrito Capital-Decreto 061 del 2003), ocasiona un alto riesgo de inundación.

La trama urbana está originada por la mezcla de urbanizaciones incompletas regulares; se organiza en manzanas de figuras rectangulares y cuadradas, que conforman la estructura de la cuadrícula, el sector con tejido espontáneo (irregular), se observan manzanas con diferentes formas similares al cuadrado y al rectángulo, con lados ondulados e inclinados. Este tejido espontáneo está localizado al noreste del barrio las Acacias, es una zona de alto riesgo de inundación por tener como borde el rio Tunjuelito de cauce abierto y nace en el embalse de la Regadera, e igual que el borde ubicado en el sur-oeste de la UPZ, amenazada por la quebrada el Tropezón. La distribución de los tamaños de las manzanas está acoplada al conjunto de viviendas unifamiliares de estrato bajo, con equipamientos propios (parques $y$

\footnotetext{
4 Urbanización incompleta: son sectores periféricos no consolidados, en estratos 1 y 2 , de uso residencial predominante con deficiencias en su infraestructura, accesibilidad, equipamientos y espacio público (Secretaria de planeación 2009).

5 Ronda hidráulica: de un cuerpo de agua, como zona de protección ambiental e hidráulico no edificable de uso público, constituida por una franja paralela o alrededor de los cuerpos de agua, medida a partir de la línea de mareas máximas (máxima inundación), Departamento Técnico Administrativo del Medio AmbienteDAMA (2004)
}

equipamiento colectivo), y zonas comerciales. Las manzanas y sus diferentes tamaños están integradas por figuras rectangulares de $105 \mathrm{~m}$ de largo por $40 \mathrm{~m}$ de ancho, con variaciones de $95 \mathrm{~m}$ de largo (frente) por $37 \mathrm{~m}$ de laterales (fondo); el tamaño promedio por manzanas es de $4.197 \mathrm{~m}$, y las manzanas de figuras cuadradas casi iguales de $48 \mathrm{~m}$ de largo por $47 \mathrm{~m}$ de ancho, con tamaño promedio por manzana de $2.164 \mathrm{~m}^{2}$. Las parcelas se presentan en las manzanas con morfología rectangular su tamaño promedio es de $139 \mathrm{~m}^{2}$ y $147 \mathrm{~m}^{2}$, se observó que los lotes cuentan en frente mínimo (ancho) que varía entre $7 \mathrm{~m}$ y $7.56 \mathrm{~m}$. La longitud (fondo) de la parcela tiene una variación entre $19.60 \mathrm{~m}$ y $19.85 \mathrm{~m}$.

Los lotes que están insertados en las manzanas de formas cuadradas, su tamaño promedio es de $119 \mathrm{~m}^{2}$ y $129 \mathrm{~m}^{2}$. Se determinó que la dimensión parcelaria es de $5.20 \mathrm{~m}$ y $10.35 \mathrm{~m}$ (frente y ancho) por $14.05 \mathrm{~m}$ y $23.20 \mathrm{~m}$, en los sectores de la urbanización residencial con actividad económica de formas cartesianas (comercio en primer piso y vivienda segundo piso).

Las manzanas de la urbanización espontánea (irregular), con morfología trapezoidal contienen un tamaño promedio de $1.246 \mathrm{~m}^{2}$, con un ancho de $23.90 \mathrm{~m}$ por $48.60 \mathrm{~m}$ de un lado (1) y $53.20 \mathrm{~m}$ de lado (2) de longitud, estructurada por parcelas de diferente tamaño entre $70 \mathrm{~m}^{2}$ y $76 \mathrm{~m}^{2}$ con dimensiones promedio de $6.10 \mathrm{~m}$ y $10.50 \mathrm{~m}$ (frente o ancho) por $12.00 \mathrm{~m}$ y $12.50 \mathrm{~m}$ (fondo). Para el caso de las manzanas que se aproximan a formas rectangulares y cuadradas son de $2.556 \mathrm{~m}^{2}$, con una dimensión de $109 \mathrm{~m}$ de longitud por $23.50 \mathrm{~m}$ de ancho, con parcelas de tamaño de $50 \mathrm{~m}^{2}$ y $64 \mathrm{~m}^{2}$; sus dimensiones son de 4.80 $\mathrm{m}$ y $6.72 \mathrm{~m}$ (frente) por $11.00 \mathrm{~m}$ y $11.40 \mathrm{~m}$ (fondo).

Zona Jerusalén, totalmente desarrollada por la autoconstrucción de sus habitantes (barrio ilegal), ocupa el cuadrante comprendido entre la av. Jorge Gaitán Cortes y la av. Ciudad de Villavicencio, en sentido norte-este y desde la quebrada Trompetica y quebrada Pena Colorada hasta el zanjón El Ahorcado, en sentido sur - oeste. Esta zona ocupa casi el $42 \%$ del contexto del supersector; los barrios que lo conforman presentan la siguiente 
Guerrero, A. - Periferias espontáneas de Ciudad Bolívar, Bogotá.

morfología: comprendida entre el límite este de la UPZ, calle $68 \mathrm{~b}$ sur y la carrera 46 $a$, en esta área predominan las manzanas con tendencia al cuadrado o al rectángulo, con $88 \mathrm{~m}$ de largo (frente) por 26 de ancho (laterales), incluyendo las aceras en algunas manzanas. Aunque algunas de ellas presentan diferentes dimensiones, que oscilan entre 75 $\mathrm{m}$ y $88 \mathrm{~m}$ (los frentes) y desde $34 \mathrm{~m}$ a $26 \mathrm{~m}$ (los laterales).

El tamaño promedio de las manzanas es de $2.875 \mathrm{~m}^{2}$, la distribución de los lotes con dimensiones de frentes $8.40 \mathrm{~m}$ y $10 \mathrm{~m}, \mathrm{y}$ desde $7.58 \mathrm{~m}$ hasta $14.15 \mathrm{~m}$ de largo (fondo), presentan un tamaño promedio de $142 \mathrm{M}^{2}$.

La calle 70-s, localizada en sentido nortesur, cruza toda UPZ, lo que genera la conformación de dos fragmentos aproximadamente del mismo tamaño, con diferentes trazados, un tramo es recto, después sufre una inclinación y ondulación, origina manzanas con diferentes formas al cuadrado y el rectángulo, varias de ellas con lados irregulares, otras se aproximan al cuadrado o rectángulo, como consecuencia del reacomodo que experimenta el tejido, donde se encuentran figuras rectangulares, trapezoidales, triangulares. Se observan manzanas con lados ondulados e inclinados con un tamaño promedio de $1.330 \mathrm{~m}^{2}$, de distribución parcelaria que oscila entre 7.65 $\mathrm{m}$ y $8.50 \mathrm{~m}$ de ancho (frente) y $13.80 \mathrm{~m}$ y $16.02 \mathrm{~m}$ de longitud (fondo), con tamaño promedio parcelar de $148 \mathrm{~m}^{2}$. También existen manzanas dispuestas en fajas en las que el ancho es el largo (fondo) del lote, el tamaño de las manzanas es de $785 \mathrm{~m}^{2}$ promedio, con una dimensión de $56 \mathrm{~m}$ de frente por $15.42 \mathrm{~m}$ de fondo, y la distribución parcelaria es de $5.90 \mathrm{~m}$ y $6.05 \mathrm{~m}$ de ancho (frente) por $13.34 \mathrm{~m}$ y $15.42 \mathrm{~m}$ de largo (fondo), y su tamaño promedio es de 96 $\mathrm{m}^{2}$.donde la Zona Ismael Perdomo, ocupa una franja norte de la localidad de Ciudad Bolívar, su tejido urbano regular e irregular (caótico), limita con la av. norte-quitosur, en sentido norte, con la av. Ciudad de Villavicencio hacia el este, con la transversal 50 por el lado sur, y con la carrera $75-\mathrm{g}$, el uso del suelo de carácter residencial, industrial, y dotacional, ocupa el $45 \%$ del supersector.
La av. Bosa, es una vía que atraviesa y comunica el sector norte con el sector sur de la UPZ, cristalizando el territorio en dos fragmentos, de un extremo se encuentra la geometría cartesiana reflejada en tramas urbanas conformada por las cuadriculas y el otro extremo está integrado por la geometría fractal que es producto de la autoconstrucción de sus habitantes (espontánea), proporcional en tamaño del territorio, originando diferentes morfologías de manzanas influenciada por los diferentes trazados de las vías con tramos rectos, ondulados e inclinados.

El sector cuenta con una morfología rectangular, marcada por rejillas cartesianas ubicadas en la parte norte y este, con los barrios la Estación, Perdomo Alto y Galicia con vocación de tipo residencial consolida y dotacional colectiva. Se observaron manzanas rectangulares, con diferentes tamaños que parten desde $2.090 \mathrm{~m}^{2}$, con una dimensión de frente de $90 \mathrm{~m}$ por 24 $\mathrm{m}$ de largo, su estructura parcelaria cuenta con dimensiones que parte desde $6.00 \mathrm{~m}$ hasta $6.40 \mathrm{~m}$ de ancho (frente) por 11.70 $\mathrm{m}$ y $12.20 \mathrm{~m}$ de longitud (fondo), para un tamaño promedio de $80 \mathrm{~m}^{2}$.

El fragmento oeste y sur, está conformado por tejido urbano irregular con diferentes morfologías: trapezoidal, onduladas e inclinadas, producidas por un trazado inclinado y curvas monstruosas que se reflejan en las manzanas urbanas y en la distribución de las parcelas. Los barrios existentes en esta zona se caracterizan por ser áreas de alto, medio o bajo riesgo de deslizamiento de tierra (remoción en masa) y riesgo de inundación ocasionada por la quebrada Santa Rita, cuya ronda hidráulica fue invadida por construcciones espontaneas con manzanas irregulares influenciadas por las formas onduladas e inclinadas. Manzanas irregulares, están marcadas por diferentes tamaños que oscilan entre $2.062 \mathrm{~m}^{2}$ hasta $3.750 \mathrm{~m}^{2}$, su estructura parcelaria contiene diferentes dimensiones de ancho desde 5.70 $\mathrm{m}$ hasta $8.40 \mathrm{~m}$ (frente), por un largo de $12.40 \mathrm{~m}$ y $14.80 \mathrm{~m}$ (fondo), para un tamaño promedio de $103,5 \mathrm{~m}^{2}$. (Ver tabla 1 ). 
Procesos Urbanos N³ Enero - Diciembre; 2016

Tabla. 1. Supersector $\left(12.75 \mathrm{~km}^{2}\right)$. Clasificación, cuantificación y distribución de las zonas. Tamaños promedios de manzanas y parcelas.

\begin{tabular}{|c|c|c|c|c|c|}
\hline Súper-sector \% & D/manzana & T/p/manzana & no manzana & D/ parcela & $\mathrm{T} / \mathrm{p} /$ parcela \\
\hline \multirow{4}{*}{$\begin{array}{c}\text { Zona San Francisco } \\
13 \%\end{array}$} & $105 \mathrm{~m} \times 40$ & \multirow{4}{*}{$2.536 \mathrm{~m}^{2}$} & \multirow{4}{*}{510} & $7 \times 19.85$ & \multirow{4}{*}{$103.25 \mathrm{~m}^{2}$} \\
\hline & $48 \times 47$ & & & $5,20 \times 23,20$ & \\
\hline & e & & & $6,10 \times 12,50$ & \\
\hline & $\mathrm{e}$ & & & $6,72 \times 11,40$ & \\
\hline \multirow{3}{*}{$\begin{array}{c}\text { Zona Jerusalén } \\
42 \%\end{array}$} & $88 \times 26$ & \multirow{3}{*}{$688.17 \mathrm{~m}^{2}$} & \multirow{3}{*}{907} & $10 \times 14,15$ & \multirow{3}{*}{$129 \mathrm{~m}^{2}$} \\
\hline & $\mathrm{e}$ & & & $8,50 \times 16,06$ & \\
\hline & $56 \times 15,42$ & & & $6,05 \times 15,42$ & \\
\hline \multirow{2}{*}{$\begin{array}{l}\text { Zona Ismael } \\
\text { Perdomo } \\
45 \%\end{array}$} & $90 \times 24$ & \multirow{2}{*}{$2.920 \mathrm{~m}^{2}$} & \multirow{2}{*}{1391} & $6,40 \times 12,20$ & \multirow[b]{2}{*}{$103.5 \mathrm{~m}^{2}$} \\
\hline & e & & & $8,40 \times 14,80$ & \\
\hline
\end{tabular}

Fuente: elaboración propia a partir de Pineda, Ferrer (2000).

\section{RESULTADOS:}

El factor de escala y los tamaños del el supersector - UPZ 66, 69, 70.

En la localidad de Ciudad Bolívar, se conformó un supersector, está integrado por tres (3) UPZ San Francisco, Jerusalén, Ismael Perdomo, estas zonas están con sus diferentes áreas o tamaños, se ordenaron de mayor a menor. (Ver tabla 2).

Tabla 2. Supersector localidad Ciudad Bolívar. Zonas y tamaños.

\begin{tabular}{cccc}
\hline UPZ & \% ocupación & Área/zona & $\begin{array}{c}\text { Tamaño/ } \\
\text { prom. }\end{array}$ \\
\hline UPZ 66 & $45 \%$ & $5,59 \mathrm{KM}^{2}$ & \\
UPZ 70 & $42 \%$ & $5,37 \mathrm{KM}^{2}$ & $4,25 \mathrm{KM}^{2}$ \\
UPZ 69 & $13 \%$ & $1,79 \mathrm{KM}^{2}$ & \\
\hline \multicolumn{4}{c}{ Área total } \\
\hline
\end{tabular}

Fuente: elaboración propia a partir de Pineda, Ferrer (2000).

A partir del tamaño del supersector $a=12.75$ $\mathrm{km}^{2}$, se averiguaron, aplicando factores de escala decrecientes, los resultados que concedían ${ }^{6}$ o se aproximaban ${ }^{7}$, al verdadero tamaño del Supersector UPZ, de la localidad en Ciudad Bolívar. De ser así, se muestra una

\footnotetext{
6 Coincidencia: Ajustarse una cosa con otra; confundirse con ella, ya por superposición, ya por otro medio cualquiera. DRAE (2014).

7 Aproximación: Máxima diferencia posible entre un valor obtenido en una medición o cálculo y el exacto desconocido. DRAE (2014).

estructura jerarquizada que está conformada por patrones fractal. Para medir las UPZ, se realizó un esquema que determina los tamaños mediante diagramas de Venn.

Al encontrar que las UPZ están fragmentadas, se construyeron sumatorias gráficas a partir del área que ocupa cada zona, de esta manera visualizar los tamaños y fijar porcentaje que ayuda a determinar con cierta precisión las áreas de las UPZ. Se utilizó un factor de escala con la progresión: $\mathrm{f}^{1} \mathrm{a}, \mathrm{f}^{2} \mathrm{a}, \mathrm{f}^{3} \mathrm{a}$; colocando tres números (valores) fraccionarios que están sujetos a la distancia del observador ( $f$ ) que serán: $1 / 2,1 / 3$, y $1 / 4$, esto se puede observar en la tabla 3.

Aplicando el factor de escala fraccionario (1/2) sobre el Supersector UPZ $\left(12,75 \mathrm{~km}^{2}\right)$, siguieron tres (3) tamaños en orden decreciente, se observó que ninguno de los valores coincide ni aproximándose a los tamaños reales de las UPZ generado por la lectura del plano. Los resultados producto de los tamaños de las UPZ San Francisco, Jerusalén, Ismael Perdomo están dentro del orden decimal, pero no existe aproximación.

Al trabajar con el factor $1 / 2$, los tamaños no responden al comportamiento fractal matemático, y estos pueden decrecer hasta el infinito. Dado que se realizó una investigación para determinar la existencia de estructura con jerarquía de escalas decrecientes (patrón fractal), donde se desarrolló el factor hasta tercera potencia, existen resultados que están cerca al tamaño real, de continuar hasta el 
Guerrero, A. - Periferias espontáneas de Ciudad Bolívar, Bogotá.

Tabla 3. Supersector. El factor de escala y los tamaños.

\begin{tabular}{|c|c|c|c|c|}
\hline $\mathbf{a}$ & Tamaño supersector & Tamaño/UPZ. & UPZ & $\begin{array}{c}\text { Tamaño promedio / } \\
\text { zona }\end{array}$ \\
\hline$(1 / 2)^{1} \times 12,75 \mathrm{~km}^{2}$ & $6.3750 \mathrm{~km}^{2}$ & $5,59 \mathrm{~km}^{2}$ & Ismael Perdomo & \\
\hline$(1 / 2)^{2} \times 12,75 \mathrm{~km}^{2}$ & $3,1875 \mathrm{~km}^{2}$ & $5,37 \mathrm{~km}^{2}$ & Jerusalén & $4,25 \mathrm{~km}^{2}$ \\
\hline$(1 / 2)^{3} \times 12,75 \mathrm{~km}^{2}$ & $1,5937 \mathrm{~km}^{2}$ & $1,79 \mathrm{~km}^{2}$ & San Francisco & \\
\hline$(1 / 3)^{1} \times 12,75 \mathrm{~km}^{2}$ & $4,2500 \mathrm{~km}^{2}$ & $5,59 \mathrm{~km}^{2}$ & Ismael Perdomo & \\
\hline$(1 / 3)^{2} \times 12,75 \mathrm{~km}^{2}$ & $1,4166 \mathrm{~km}^{2}$ & $5,37 \mathrm{~km}^{2}$ & Jerusalén & $4,25 \mathrm{~km}^{2}$ \\
\hline$(1 / 3)^{3} \times 12,75 \mathrm{~km}^{2}$ & $0,4722 \mathrm{~km}^{2}$ & $1,79 \mathrm{~km}^{2}$ & San Francisco & \\
\hline$(1 / 4)^{1} \times 12,75 \mathrm{~km}^{2}$ & $3,1875 \mathrm{~km}^{2}$ & $5,59 \mathrm{~km}^{2}$ & Ismael Perdomo & \\
\hline$(1 / 4)^{2} \times 12,75 \mathrm{~km}^{2}$ & $0,7968 \mathrm{~km}^{2}$ & $5,37 \mathrm{~km}^{2}$ & Jerusalén & $4,25 \mathrm{~km}^{2}$ \\
\hline$(1 / 4)^{3} \times 12,75 \mathrm{~km}^{2}$ & $0,19921 \mathrm{~km}^{2}$ & $1,79 \mathrm{~km}^{2}$ & San Francisco & \\
\hline
\end{tabular}

Fuente: elaboración propia a partir de Pineda, Ferrer (2000).

infinito, se encontrarían escalas no manejables para el estudio urbanístico.

Con el factor de escala (1/3) se aplicaron tres (3) valores decrecientes en relación con el tamaño del Supersector $\left(12,75 \mathrm{~km}^{2}\right)$, ninguno de los valores coincide ni se aproxima a las áreas reales de las UPZ generadas por la lectura del plano. Al aplicar el factor (1/3), las aéreas no corresponden a un comportamiento fractal matemático, pudiendo decrecer indefinidamente; se desarrolló hasta la tercera potencia, para alcanzar tamaños próximos al tamaño del Supersector.

Con el factor de escala (1/4), se obtuvieron tres (3) tamaños en orden decreciente, no coinciden ni se aproximan a ningún tamaño real de las UPZ de los que se midieron en el plano. Como resultado se generaron diferencias mínimas (decimales), pero ninguna se encuentra entre las condiciones de aproximación y/o coincidencia.

En cuanto a la estructura del Supersector, las áreas y su distribución, después de caracterizar las UPZ de la localidad de Ciudad Bolívar, y utilizados los factores de escala al tamaño del Supersector, se estableció en forma teórica la secuencia decreciente ${ }^{8}$ de las UPZ y se comprobó que no existen patrones fractales en ninguna zona. Con los resultados se analizó la distribución de los tamaños, buscando la existencia de relaciones fractales; para esto se usó la regla de escala y se aplicó la ley de potencias.

8 Decreciente: que decrece (R.E.A 2014).
Esta investigación demandó la precisión de estructuras y valores, por esta razón se estableció que el área del Supersector sería el valor de referencia $\left(\mathrm{km}^{2}\right)$, después allí se identificaron en forma decreciente los tamaños de la UPZ según su propiedad. A su vez, la UPZ están conformadas por asentamientos espontáneos (ilegales), que se encuentran fragmentados dentro de la localidad de Ciudad Bolívar.

Para realizar la operación, la constante es 1,5 dimensiones fractal, el cual es el promedio entre 1 y 2 de la dimensión en fractales, y factor de escala un número fraccionario ${ }^{9}$ igual a (1/2); para la distribución del tamaño se utilizó la ley potencia:

$\mathrm{q}_{\mathrm{e}=\mathrm{T}(\mathrm{Zn})^{-\mathrm{d}}}$

$T=$ tamaño mayor; $z=$ tamaño promedio; $\mathrm{d}=$ dimensión fractal $(1,5) ; \mathrm{f}=$ factor de escala $(1 / 4) ; e=$ numero entero que representan la escala.

$\mathrm{q}=$ número de unidades;

$\mathrm{K}=$ constante

Sustituyendo, tenemos que:

$\mathrm{q}=3^{(1,5) \cdot \mathrm{n}} \cdot \mathrm{T}$, donde $\mathrm{T}=1$

Si e $=0$ resulta $\mathrm{q}_{0}=2^{(1,5) \cdot 0}=2^{0}=1,0000$ localidad $\mathrm{e}=1 \mathrm{q}_{1}=2^{(1,5) \cdot 1}=2^{1,5}=2,8284$ supersector $\mathrm{e}=2 \mathrm{q}_{2}=2^{(1,5) \cdot 2}=2^{3}=8,0000$ UPZ $\mathrm{e}=3 \mathrm{q}_{3}=2^{(1,5) \cdot 3}=2^{4,5}=23,0000$ barrios

9 Numero Fraccionario: la dimensión efectiva es la dimensión fraccional que representa el grado de irregularidad o discontinuidad de un objeto, que se relaciona a la morfología de la naturaleza que se repite a diferente escala (Ugas 2010). 
Al confrontar los resultados productos de la ecuación de distribución, con el número de unidades cuantificadas en el plano. (Ver tabla 4).

Tabla 4. Comparación de los resultados teóricos con la realidad del supersector.

\begin{tabular}{lcccc}
\hline Tamaños & $\begin{array}{c}\text { Escala Fact/ Unidad } \\
\text { e }\end{array}$ & esc & $\begin{array}{c}\text { Unidades } \\
\text { q }\end{array}$ & $\begin{array}{c}\text { existentes } \\
\text { supersector }\end{array}$ \\
\hline Localidad & 0 & $1 / 3$ & 1 & 1 \\
Supersector & 1 & $1 / 3$ & 3 & 2.82 \\
UPZ & 2 & $1 / 3$ & 8 & 8 \\
Barrios & 3 & $1 / 3$ & 127 & 23 \\
\hline
\end{tabular}

Fuente: elaboración propia a partir de Pineda, Ferrer (2000).

Las unidades (q): $1,3,8$ que fueron las resultantes de la ley de potencia al aplicar el factor $(f=1 / 2)$ en diferentes escalas decrecientes: $0,1,2$, y 3 (cada vez que aumenta el entero disminuye la escala), concuerdan con las unidades existentes en los planos de las UPZ de la localidad Ciudad Bolívar. Se pudo establecer coincidencia con la cantidad de la localidad identificada como Ciudad Bolívar, también existe aproximación con la cantidad de UPZ que integran el Supersector, y por último, hay coincidencia con la cantidad de UPZ que conforman la localidad de ciudad bolívar, lo que significa que se cumple con la ley de potencia en la distribución de los tamaños o áreas de las zonas; se puede establecer que el Supersector contiene una estructura morfológica de comportamiento decreciente (fractal).

\section{REFLEXIONES FINALES:}

Las conclusiones de esta disertación, están encaminadas a la creación del cómo la teoría de los fractales, puede determinar la necesidad de robustecer los planes urbanos o la renovación urbana (cirugías urbanas) de las UPZ de la localidad.

El Supersector: está integrado por tres (3) UPZ San Francisco, Jerusalén, Ismael Perdomo, son asentamientos espontáneos producto de migración, que experimentan un crecimiento a partir del casco central de la ciudad de Bogotá el cual está estructurado por una cuadrícula cartesiana, que sufre una metamorfosis en su morfología, producto del crecimiento de las periferias internas espontáneas y la topografía inclinada que ocasiona una trama irregular, que se conecta a la trama inicial (regular) por diferentes puntos estableciendo secuencias, pero con una fragmentación geométrica.

El factor de escala: en el supersector (UPZ San Francisco, Jerusalén, Ismael Perdomo), se le aplicó tres factores fraccionarios consecutivos $(1 / 2,1 / 3,1 / 4)$ al tamaño de supersector. Ninguno de los tres tamaños de la UPZ que integran el supersector, ninguno de los valores coinciden ni se aproximan al área real resultante del plano. Esto es indicativo que las UPZ no están inscritas a la jerarquía de escala, y se recomienda realizar una cirugía urbana (renovación urbana) de estas UPZ, para poder terminar con la fragmentación de la ciudad de Bogotá, y comenzar a construir una urbe compacta, la cual se recomienda como base para el desarrollo sustentable.

Tamaños y distribución, en el supersector: el área promedio que representa las manzanas es de $1.612,08 \mathrm{~m}^{2}$, al cotejarlo con la superficie de las UPZ, aplicando los factores $1 / 2,1 / 3$ y $1 / 4$, se pudo observar un comportamiento decreciente. (Ver figura 1).

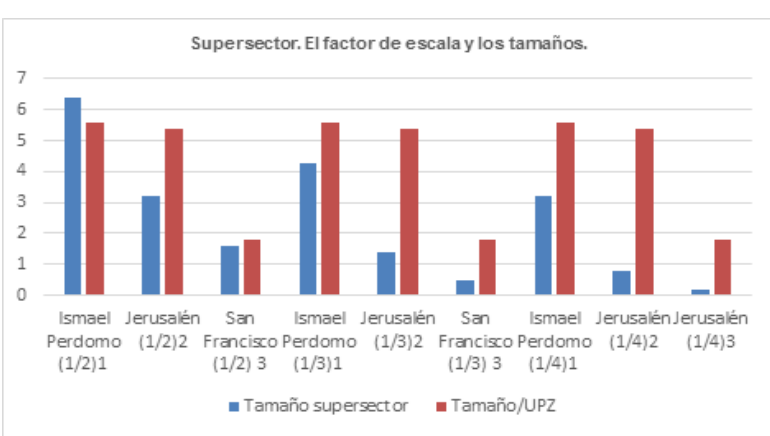

Figura 1. Comparación de los resultados del factor de escala y los tamaños.

Fuente: elaboración propia.

Se estableció que la dimensión promedio de las manzanas es: frente $105 \mathrm{~m}$, fondo $47 \mathrm{~m}$. El tamaño del lote, de la UPZ San Francisco es de $103,25 \mathrm{~m}^{2}$, Jerusalén $129 \mathrm{~m}^{2}$, e Ismael Perdomo $103 \mathrm{~m}^{2}$. 
Guerrero, A. - Periferias espontáneas de Ciudad Bolívar, Bogotá.

Se puede establecer que las periferias internas espontáneas ubicadas en las UPZ San Francisco, Jerusalén, Ismael Perdomo de Ciudad Bolívar, se caracterizan por los siguientes patrones:

Los habitantes de los asentamientos irregulares, cuentan con redes sociales muy bien interconectadas y consolidadas de talento en diseño urbano y la energía de construcción fundamentadas por el sentido de pertenencia.

Cada barrio de las UPZ, se identifica por contener su propio trazado, lo que genera una variedad de discursos urbanos que se reflejan en la variedad de dimensiones y figuras geométricas en manzanas.

La morfología es conformada por geométricas orgánicas, que representan las diferentes dinámicas sociales, económicas, culturales, políticas y urbanas de sus habitantes, es por este aspecto que es rechazada por los gobiernos, es un comportamiento fractal.

\section{Presentan diferentes formas de apropiación parcelaria.}

La densidad de la población es muy alta, lo que representa lotes con dimensiones pequeñas y un aprovechamiento al máximo. Existen manzanas localizadas en los alrededores de la ronda hidráulica protectora de las diferentes quebradas, las que la colocan en alto riesgo de inundación (áreas no urbanizables).
La infraestructura de servicios públicos es de regular funcionamiento, debido a que en la actualidad existen barrios que no cuentan con los servicios públicos mínimos (agua, alcantarillado, teléfono).

Los equipamientos que existen en las UPZ, son pocos para la cantidad de habitantes.

\section{RECOMENDACIONES}

Se sugiere seguir profundizando las posibilidades que brinda la nueva ciencia, abordando en conjunto, las tres dimensiones: longitud, área y volumen. Esto implica adicionar el empleo de ecuaciones diferenciales con el apoyo del computador.

Se recomienda para los proyectos de renovación urbana, contar con soluciones de transporte público de calidad y no contaminantes, la cual iría en beneficio de todos los ciudadanos; de esta manera se encontrará la integración urbanística de los barrios y se aumentarán las oportunidades de sus habitantes.

\section{FUTURAS INVESTIGACIONES}

Se propone para una próxima investigación, realizar la comparación entre los imaginarios colectivos populares y los patrones fractales de construcción de asentamientos espontáneos en Latinoamérica.

\section{REFERENCIAS.}

Alcaldía Mayor de Bogotá. (2003). Plan de gestión ambiental del Distrito Capital. Decreto 61 de 2003.

Borie, A. Micheloni, P. Pinon, P. (2008). Forma y deformación. Editorial: Reverte. Barcelona. Ferrer Y Arroyo, M (2012). Gobernar la complejidad urbana desde la sostenibilidad. Evaluación del gobierno de la gestión visible utilizando indicadores de gobernanza (Venezuela). Tesis doctoral. Escuela Técnica Superior de Ingenieros Industriales. Universidad Politécnica de Madrid. España.

Gutiérrez, L. (2009). Ciudad informal: la historia de un barrio ilegal. Editorial: Uniandes. Bogota. 


\section{Procesos Urbanos Nº 3 Enero - Diciembre; 2016}

Lynch, K. (2012). La imagen de la ciudad. Editorial: Gustavo Gili. Barcelona.

Leal, N. (2000). El método fenomenológico: principios, momentos y reducciones. Revista de Investigación Científica, Humanística y Tecnológica Digital. Universidad Nacional Abierta n5. Vol 1. Caracas.

Moisset, Inés. (2003). Fractales y formas arquitectónicas. Editorial: División Editorial. Argentina.

Moisset, I. (2001). El desafío de los fractales. Revista Summa+, n051. España.

Morín, E. (2011). La vía para futuro de la humanidad. Editorial: Paidós. Madrid - España.

Morín, E. (2005). Introducción al pensamiento complejo. Editorial: Gedisa. Barcelona- España. Plan de Ordenamiento Territorial de Bogotá. (2013). Decreto 364 por el cual se modifica excepcionalmente las normas urbanísticas. Disponible: http://bogotahumana.gov.co/decreto/ decreto364 2013.pdf

Pineda Paz, E. (2009). Plan urbano, morfología y fractales. Tesis doctoral no publicada. Facultad de Arquitectura y Diseño de La Universidad del Zulia, Maracaibo.

Salingaros, N. (2005). Principios de estructura urbana. Conectando la ciudad fractal. Universidad de Texas. Texas.

Salingaros, N. (2007). El futuro de las cudades. Universidad de Texas. Texas. http://www. rebelion.org/portada.php

Secretaria Distrital de Planeación. (2009). Conociendo la localidad de Ciudad Bolívar. Diagnóstico de los aspectos físicos, demográficos y socioeconómicos. Edición la Secretaria Distrital de Planeación en cumplimiento de su misión institucional, y en el marco del Decreto 550 de 2006.

Secretaria de Hacienda. (2004). Recorriendo Ciudad Bolívar. Diagnóstico físico y socioeconómico. Edición informe para garantizar el sistema financiero industrial, y de los ciudadanos.

Sola-Morales, I. (1996). Presente y futuro. La arquitectura en las ciudades. Colegio Oficial de Arquitectos de Catalunya/Centro de Cultura Contemporânea. Barcelona. http://casanchi. $\mathrm{com} / \mathrm{mat} / \mathrm{fractalesgcaos} 01 . \mathrm{htm}$

Under, J. (2007). Un recorrido por la ciudad fractal. Revista Entre Caos Digital. Disponible: http://entrecaos.wordpress.com/

Ugas, G. (2012). La complejidad un modo de pensar. Editorial: Taller permanente de estudios epistemológicos. Táchira-Venezuela.

Ugas, G. (2010). La complejidad de lo efimero. Editorial: Ediciones gema. BarquisimetoVenezuela. 